\title{
In vitro properties of concentrated canine platelets stored in two additive solutions: a comparative study
}

\author{
N. Hlavac ${ }^{1 *}$ (D, C. S. Lasta ${ }^{2}$, M. L. Dalmolin ${ }^{3}$, L. A. Lacerda ${ }^{3}$, D. de Korte ${ }^{4}$, N. A. Marcondes ${ }^{5}$, S. R. Terra ${ }^{1}$, \\ F. B. Fernandes ${ }^{6}$ and F. H. D. González ${ }^{7}$
}

\begin{abstract}
Background: Platelet transfusion therapy poses many challenges in veterinary clinical practice. Lack of readily available blood donors, short shelf-life, and inability to administer a sufficient number of platelets to meet a dog's transfusion need are the major difficulties encountered. Platelet additive solutions are already in use at American and European human blood banks, showing to be a realistic alternative. This study compares the in vitro platelet function in plasma, Composol, or SSP+ during storage for 13 days. Platelet rich plasma-platelet concentrate with 35\% plasma and $65 \%$ platelet additive solutions (Composol or SSP+) and a control group (100\% plasma) were prepared. Swirling, platelet count, blood gases, metabolic variables, platelet activation markers, and apoptosis markers were analyzed on days $1,5,9$ and 13 .

Results: Swirling was well preserved and $\mathrm{pH}$ was acceptable $(>6.2)$ during storage for all platelet additive solutions units until day 9. SSP + units showed more stable pH and metabolic variables until day 13. Platelets in plasma showed higher glucose consumption than in Composol or in SSP+. The platelet additive solutions units showed better platelet metabolism maintenance, reduced glucose consumption and lactate production. The apoptotic markers were still low for 9 days in platelet concentrates with platelet additive solutions, suggesting the possibility to extend the shelf life with the use of SSP+ or Composol.
\end{abstract}

Conclusions: Our findings suggest that the uses of Composol and SSP+ in canine platelet concentrates are potential alternatives in veterinary blood banks.

Keywords: Composol, Platelet storage lesion, SSP +, Storage solutions, Veterinary transfusion medicine

\section{Background}

One of the goals of transfusion medicine is to assure the production of quality blood components. The platelet concentrate (PC) availability is limited due to its short-term storage. During storage, platelets suffer structural and biochemical changes that are collectively called platelet storage lesions (PSL) [1]. Extending the shelf-life has effects on the quality of platelets which have been studied in PC stored for more than 5 days. For several reasons, storing platelets in platelet additive solutions (PAS) is turning into

\footnotetext{
* Correspondence: rch.nicole@gmail.com

${ }^{1}$ Clinical Pathology Laboratory, Veterinary Medicine Faculty, Universidade do Sul de Santa Catarina, Tubarão, Brazil

Full list of author information is available at the end of the article
}

a routine practice, and it is already standard in human blood banks in Europe and The United States [2].

PAS are isotonic crystalloid media containing citrate as anticoagulant and acetate as fuel for aerobic metabolism. Initially, the main motivation to use PAS was to increase the availability of plasma for the fractioning and production of derived components. However, other advantages were observed, such as being a sterile environment free of pathogens and the standardized composition in comparison to plasma from donors [3-5].

Several PAS formulations are being tested since 1980. In vivo studies with the most recently developed solutions have shown good results of platelet increment and recovery as well as the reduced occurrence of transfusion reactions in human patients [6-8]. 
Studies in human blood banks have already tested the platelet metabolism in vitro using PAS for periods exceeding 12 days $[9,10]$. In some countries in Europe, the storage timing of PC was extended to 7 days with the use of PAS [2]. There are already four PAS generations, Composol (PAS-D) and SSP+ (PAS-E), tested in this study, are from the third generation and are used to preserve PC in European blood banks [3, 6, 11].

The search for new additive solutions for PC preservation has always been a concern in human medicine, and nowadays it is also of interest for the veterinary medicine. The biochemical evaluation during canine PC storage with different PAS represents some practical information for veterinary medicine. Canine platelet metabolism has similar characteristics to the human, and the PC storage time is also a difficulty at the veterinary blood banks. The quality control requirements of canine $\mathrm{PC}$ are based on human models. Therefore, it is believed that the results concerning the viability and PSL should be similar to the already reported in vitro tests done with human PC.

This study aims to compare the parameters of in vitro platelet metabolism from concentrates of canine platelets stored in plasma and in two PAS.

\section{Methods}

A volume of $450 \mathrm{~mL}$ whole blood was collected in blood bag with $\mathrm{CPD}^{1}$ from healthy canine donors, weighing over $28 \mathrm{~kg}$. Physical examination, CBC, chemistry profile, and infectious disease screening were performed for each dog. The animals were privately owned; all owners provided their consent for their dogs to be used in this study in accordance with bioethics concepts applied to animal research. This study was approved by the Ethics Committee on Animal Use from the Federal University of Rio Grande do Sul (Approval protocol \#20528).

The experiment was carried out in three groups (Treatment 1: $100 \%$ plasma (control) $n=13$; Treatment 2: $\mathrm{SSP}_{+}{ }^{2} n=13$; Treatment 3: Composol $^{3} n=14$ ), in a total of 40 blood bags.

\section{PC preparation}

After collection, the bags were left undisturbed at room temperature for one hour and then were processed to obtain the PC through the platelet rich plasma method (PRP). The first centrifugation ${ }^{4}$ was light, $1600 \mathrm{~g}$ for $6 \mathrm{~min}$ at $22^{\circ} \mathrm{C}$.

The PRP was transferred to an empty TOTM-PVC ${ }^{1}$ bag through manual plasma extractor. ${ }^{5}$ The PRP bag was subjected to a second $\operatorname{spin}^{4}$ (3300 $g$ for $8 \mathrm{~min}$ at $22^{\circ} \mathrm{C}$ ), to remove the excess of plasma. Manual extraction ${ }^{5}$ was used and additive solutions ${ }^{2,3}$ were added in the test groups in the proportion of $65 \%$ of PAS to $35 \%$ of residual plasma as recommended by the manufacturer's (32-47\%) [12], with a final volume of about $65 \mathrm{~mL}(22 \pm 2 \mathrm{~mL}$ of plasma and $42 \pm 2 \mathrm{~mL}$ of PAS). The control group PCs $(100 \%$ plasma) were prepared in the traditional way, by manual extraction ${ }^{5}$ targeting the volume of approximately $65 \mathrm{~mL}$. The compositions of the chosen PAS are shown in Additional file 1.

A sampling site coupler $^{6}$ was placed in all bags [13]. The calculated volume of PAS was added with the aid of sterile syringe and needle. The volume of the units was calculated in accordance with the specific gravity of resuspension solution $(1.026 \mathrm{~g} / \mathrm{mL}$ for plasma, $1.006 \mathrm{~g} /$ mL PAS) [3].

After this procedure, the bags were identified and kept at rest for an hour. Afterward, they were placed in a linear platelet shaker ${ }^{7}$ located in a preservation chamber ${ }^{8}$ in regulated temperature $\left(22-24{ }^{\circ} \mathrm{C}\right)$ for 13 days.

\section{Sampling}

The PCs stored in each treatment were subjected to evaluation at established time points. The sampling was done by sampling site coupler ${ }^{6}$ using syringe and sterile needles [14].

Analyzes were carried out on days 1, 5, 9 and 13. Platelet counts, MPV, PDW, swirling, $\mathrm{pH}$, glucose, lactate, $\mathrm{LDH}, \mathrm{pO}_{2}$ and $\mathrm{pCO}_{2}, \mathrm{ATP}$, and flow cytometry to evaluate CD61, CD62P, Annexin V and JC-1 were assessed. Residual leukocyte count was performed on day 1 (24 h after collection). PCs samples were sent for microbiological culture on days 5 and 13.

\section{Qualitative and morphological variables}

Before the bag sampling, an evaluation of platelet swirling was performed. Swirling is a non-invasive method for assessing platelet viability; it is caused by light diffraction due to the alignment of normal discoid shaped platelets. In this evaluation zero indicates no swirling and 3 indicates great swirling [15]. Residual leukocyte count was performed using the Nageotte chamber. ${ }^{9}$ The MPV, PDW and platelet counts were performed on an automated hematology counter. ${ }^{10}$

\section{Surface markers and platelet function}

Identification of platelet surface markers was performed through flow cytometry with monoclonal antibodies (MoAbs) CD61 FITC, ${ }^{11}$ used for identification and quantification of platelet population; and p-selectin ${ }^{12}$ (CD62P), for evaluation of platelet activation.

\section{Gas analysis and metabolic variables}

To assess platelet metabolism, the values of bicarbonate, glucose, $\mathrm{pO}_{2}$ and $\mathrm{pCO}_{2}$ were determined through a portable gas analyzer. ${ }^{13}$ Also, in order to determine the ATP, samples were prepared in trichloroacetic acid solution, frozen at $-80{ }^{\circ} \mathrm{C}$ [12], and measured by bioluminescence assay $^{14}$ aided by multi-mode microplate reader, ${ }^{15}$ 
according to the manufacturer's instructions. The determination of lactate was performed through dry chemistry. ${ }^{16}$ The $\mathrm{pH}$ was measured by $\mathrm{pH}$ meter ${ }^{17}$ according to the manufacturer's instructions. All samples were tested in duplicate.

\section{Markers of apoptosis and mitochondrial potential}

LDH was analyzed with dry chemistry ${ }^{16}$. The exposure of PS (phosphatidylserine) was assessed with annexin V marker ${ }^{18}$ and the percentage of cells positive for the marker was quantified. Alterations in mitochondrial membrane potential $(\Delta \Psi \mathrm{m})$ were determined with $\mathrm{JC}-1^{19}$ evaluation. Relatives degrees of mitochondrial polarization were quantified by measuring the red-shifted (FL-2) JC1 aggregates, which are favored under of high membrane potential.

All flow cytometric experiments were performed on a flow cytometer ${ }^{20}$ where 30,000 events were acquired for each analysis ${ }^{20}$. Data were analyzed using FCS Express 5 software. $^{21}$

\section{Microbiological analysis}

Inoculation was performed in BHI, with aerobic and anaerobic culture at $37{ }^{\circ} \mathrm{C}$ in samples from days 5 and 13 of assessment.

\section{Statistical analysis}

The quantitative data were expressed as the mean \pm standard deviation. Two-way ANOVA was performed to analyse the effects of PAS and plasma. One-way ANOVA was performed to analyse the residual WBC count. When indicated, a post-hoc Tukey test or Duncan's multiple range test were performed. The analyses were performed through Graph Pad Prism 6.0. ${ }^{22}$ The results with $p<0.05$ were considered significant. Pearson correlation between variables was performed, considering significant values of $p<0.05$.

\section{Results}

\section{Composition of platelet concentrates}

In vitro quality parameters were evaluated on day 1 (Table 1). All PCs had leukoreduction, average volume,
$\mathrm{pH}$, swirling and platelet concentration according to the quality control recommendations of regulatory authorities [16], and there was no significant difference between treatments. Three bags were excluded due to a contamination by Staphylococcus spp. negative coagulase on days 5 (one from each PAS) and 13 (Composol unit).

\section{In vitro evaluation during the stock}

Platelet count remained stable over storage time and there was no difference between plasma and PAS groups (Additional file 2). Regarding the swirling, a significant reduction in this parameter was observed over time $(p<$ 0.001). The swirling was kept $2+$ (suitable for use) until day 9 in both PAS, and until day 13 in the PC stored with SSP+ (Fig. 1).

The PDW and MPV morpho-structural rates showed differences between the days of evaluation. Both parameters had a significant increase in days 5, 9 and 13 compared to day $1(p<0.001)$ (Fig. 1$)$, and there was a significant PDW increase in the control group on day 13 $(p<0.001)$ compared to the PAS groups. There was no significant difference in platelet count and labeling with CD61 along time or between groups. The percentage of CD61 positive cells remained above 85\% (Additional file 2 ). The platelet activation evidenced by CD62P was higher in the control group compared to PAS $(p<0.001)$, with no difference among the PAS groups. There was an increased CD62P on day1 $(p=0.014)$ in all treatments, and on day 13 in the control group $(p<0.001)$.

On day 1 , the control group showed higher $\mathrm{pH}$ values in comparison to the PAS groups (Fig. 1), however, the control group showed significant decrease along the storage time. Meanwhile in Composol and SSP+ there was an increase of $\mathrm{pH}$ during long-term storage. In Composol solution, $\mathrm{pH}$ was below the acceptable $(<6.2)$ on days 5, 9 and 13. The units held in SSP+ showed a more stable $\mathrm{pH}$ during the 13 days of storage (Fig. 1, Additional file 2).

A decrease in ATP concentration was observed in all groups, there was no difference between the treatments (Fig. 1). A decline of glucose consumption and an increased lactate production along the time was observed

Table 1 Residual leukocyte, platelet counts, swirling and pH on the experimental day 1

\begin{tabular}{llll}
\hline & Day 1 & & Composol \\
\cline { 2 - 4 } & Plasma & SSP+ & $63.48 \pm 3.11$ \\
\hline Volume $(\mathrm{ml})$ & $65.92 \pm 1.977$ & $64.05 \pm 2.66$ & $0.005 \pm 0.002$ \\
Residual leukocytes $\left(\times 10^{6} /\right.$ unit) & $0.0037 \pm 0.0021$ & $0.010 \pm 0.009$ & $7.64 \pm 2.44$ \\
Platelet count $\left(\times 10^{10} /\right.$ unit) & $7.197 \pm 2.86$ & $7.88 \pm 2.13$ & $2.86 \pm 0.53$ \\
Swirling & $3 \pm 0$ & $3 \pm 0$ & $6.44 \pm 0.45 \mathrm{~b}$ \\
$\mathrm{pH}\left(22^{\circ} \mathrm{C}\right)$ & $7.162 \pm 0.24 \mathrm{a}$ & $6.72 \pm 0.20 \mathrm{~b}$ & \\
\hline
\end{tabular}

Different lowercase letters represent significantly different values $(p<0.05)$ between treatments. Results reported as mean \pm standard deviation 

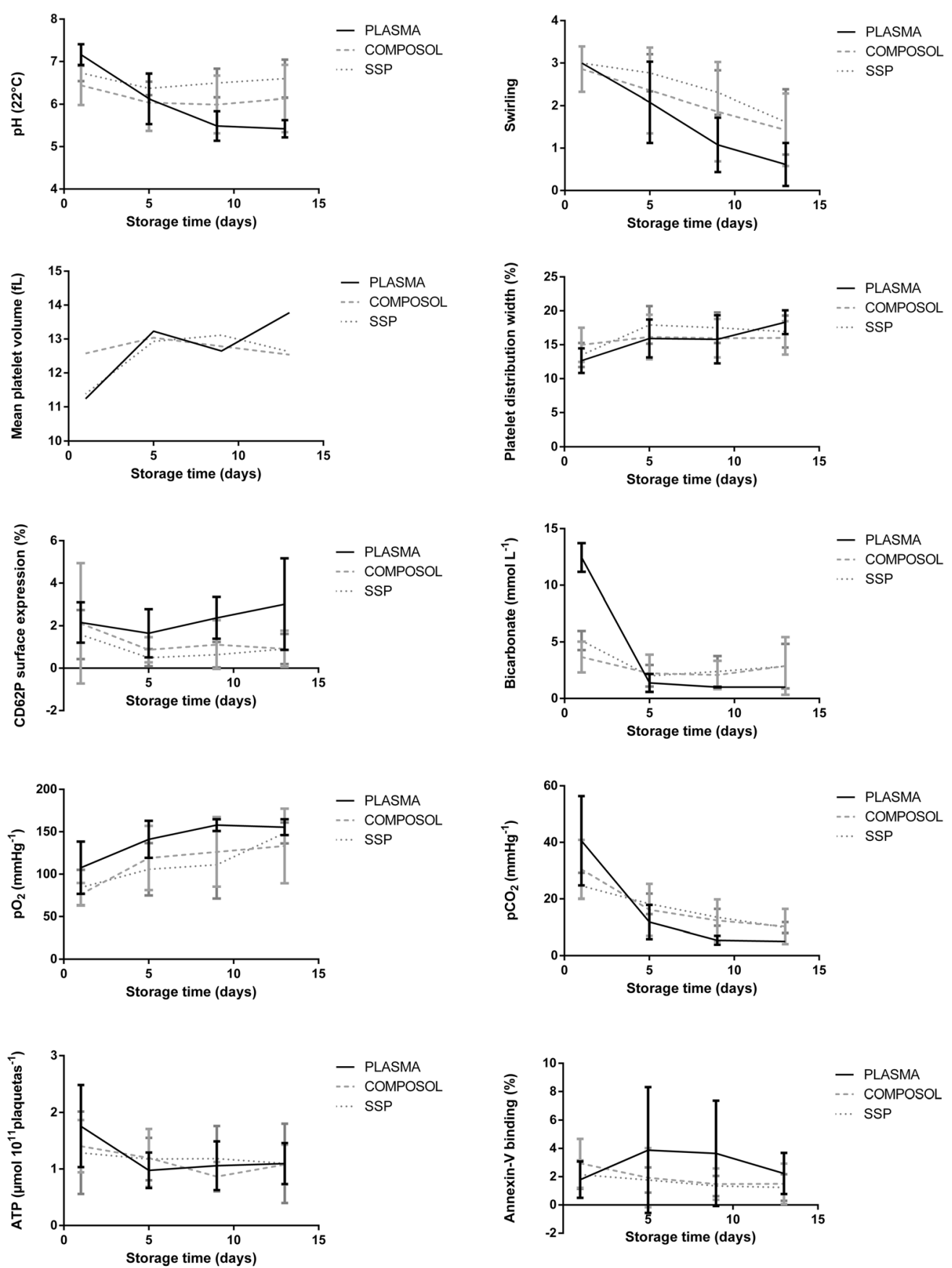

- PLASMA

-.. COMPOSOL SSP
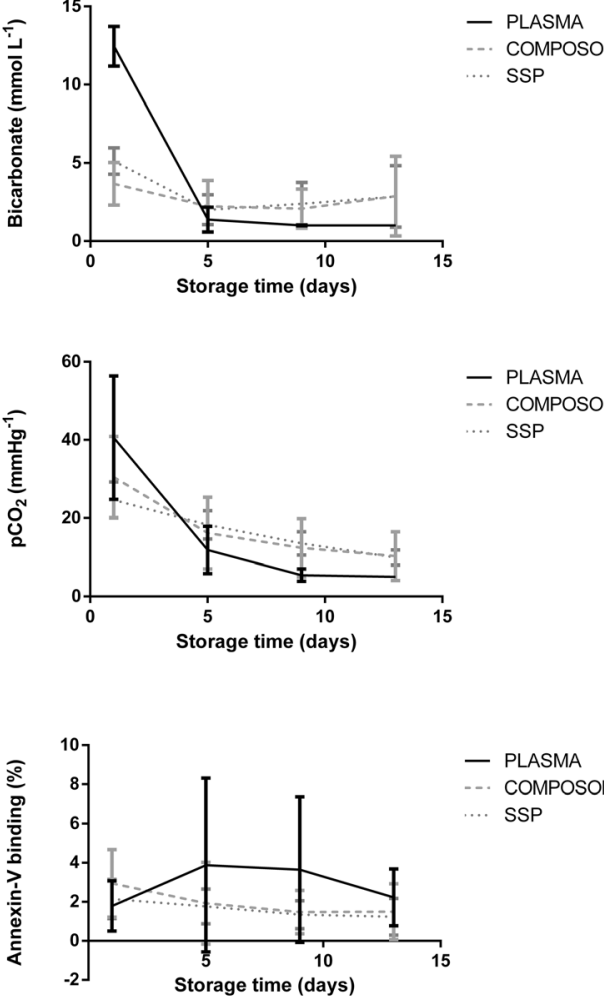

-. COMPOSOL

SSP

\section{- PLASMA}

SSP

-. COMPOSOL

SSP

-.. COMPOSOL

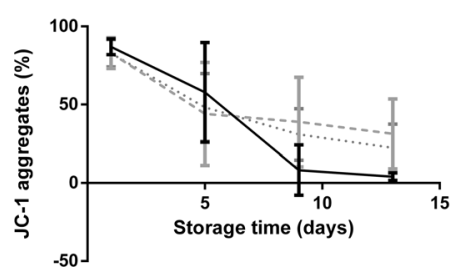

- PLASMA - COMPOSOL SSP

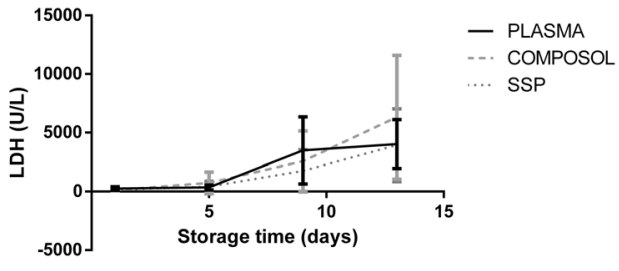

Fig. 1 Linear regression graphs of the in vitro parameters of plasma platelet concentrates and additive solution, stored for 13 days 
$(p<0.001)$, and the average of consumption/production was significantly higher in the control group compared to the PAS groups $(p<0.001)$ (Figs. 2 and 3, Additional files 3 and 4).

There was a decrease in bicarbonate concentration during the storage time $(p<0.001)$. The initial bicarbonate concentration was higher in the control group $(p<$ 0.001 ), the decrease in concentration was consequently higher in this group compared to PAS (Fig. 1). A reduction in $\mathrm{pCO}_{2}$ was observed during the storage time $(p<$ 0.001 ) and the $\mathrm{pO}_{2}$ behaved inversely over the storage time in all groups $(p=0.001)$ (Fig. 1).

There were no alterations in PS throughout the storage time, and there was a higher expression in the control group compared to SSP+ $(p=0.007)$ and Composol $(p=0.050)$ (Fig. 1). The LDH activity showed an increase over time in all groups (Fig. 1), being significant from the 9th day of storage, without differences between the groups $(p<0.001)$ (Fig. 1).

Regarding the $\Delta \Psi \mathrm{m}$, there was a decrease in the percentage of JC-1 aggregates over the storage time $(p<$ 0.001 ), leveling of between days 9 and 13. In the last 2 days of assessment, there was a significant lower percentage of JC- 1 aggregates PC in the control group ( $p<$ 0.001) (Fig. 1).

\section{Correlation study}

The results of the correlations (Table 2) emphasize the findings described before, and are closely related to aerobic and anaerobic metabolism performed by platelets in the stock period. A decrease in glucose, $\mathrm{pCO}_{2}$, $\mathrm{HCO}_{3}, \mathrm{ATP}, \mathrm{pH}$ and $\Delta \Psi \mathrm{m}$ was observed along time; and the data show a positive correlation between these parameters. Also, a positive correlation interposes between the lactate production, cytoplasmic LDH release, increase of $\mathrm{pO}_{2}$ and increased morpho-structural parameters - MPV and PDW.

\section{Discussion}

This is the first study to assess the feasibility to store canine platelets with PAS obtained by PRP method. The comparative study of PAS revealed that both solutions maintained the in vitro quality of canine platelets for up to 9 days after collection. Parameters such as swirling, $\mathrm{pH}$ and platelet counts were maintained for up to 13 days with the use of SSP+.

The results of metabolic evaluation, cell death, mitochondrial membrane polarization and activation support the in vitro stability evidenced in this study. The control group also remained stable until the fifth day, as expected, except for the average $\mathrm{pH}$ value $(6.1 \pm 0.59)$. The values recommended by regulatory authorities and studies in humans and canines were taken as a comparative basis. When canine PC parameters in plasma and PAS produced by PRP method - were compared, similar data to those produced by buffy-coat method (BC) or apheresis in humans were observed. However this is the first study that report PC viability in PAS produced by PRP method (Table 3) [3, 14, 16-22].

All PAS require residual plasma to metabolism maintenance. The experiences of American and European studies indicate that when using PAS with less than $20 \%$ residual plasma through the $\mathrm{BC}$ method, there will be difficulty in obtaining blood component with the desired quality. Therefore, researchers suggest a residual plasma volume of $32-47 \%[8,11,12]$. Our study showed adequate platelet parameters using $35 \%$ of residual plasma with manual extraction technique, suggesting that the technique is feasible and easy to execute.

Adequate platelet count is one of the most important pieces of information for clinicians, so it is a crucial point when producing a PC [23]. Studies that evaluated canine PC in $100 \%$ plasma observed a significant reduction of platelet count, it was associated with platelet activation and formation of microaggregates, fragmentation or loss of platelet integrity, and these may be the reasons of reduction of platelet count during the storage period $[20,21]$. Our results are in agreement with previous studies evaluating human $\mathrm{PC}$ in $\mathrm{SSP}+$ and Composol, where no variation in platelet count was observed [3, 15, 19]. Van der Meer (2012) stated difference in this parameter, the authors suggest that the low
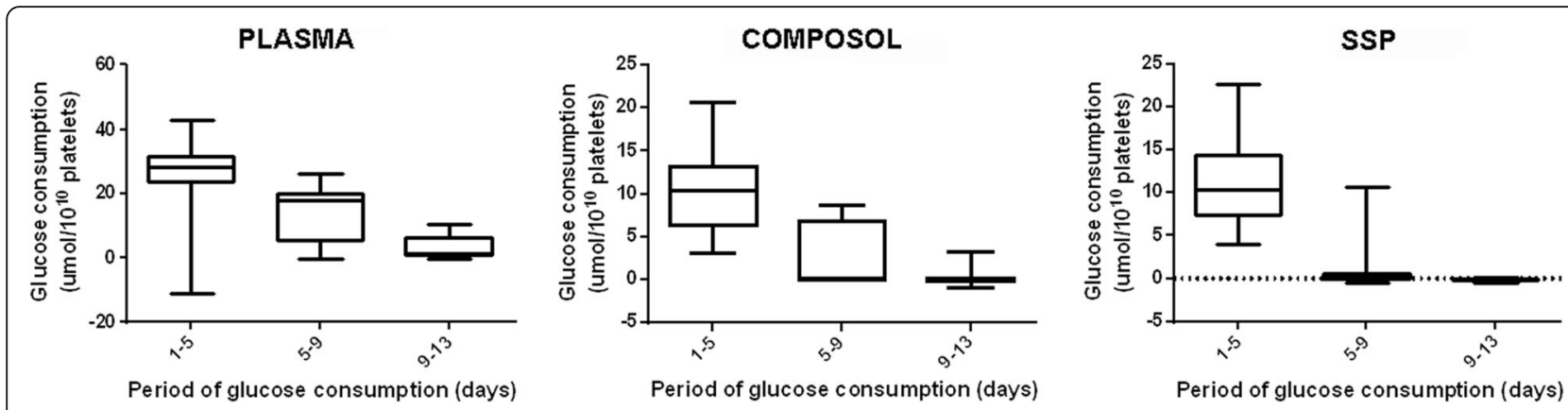

Fig. 2 Glucose consumption rates of platelet concentrates stored in plasma and additive solution for 13 days 

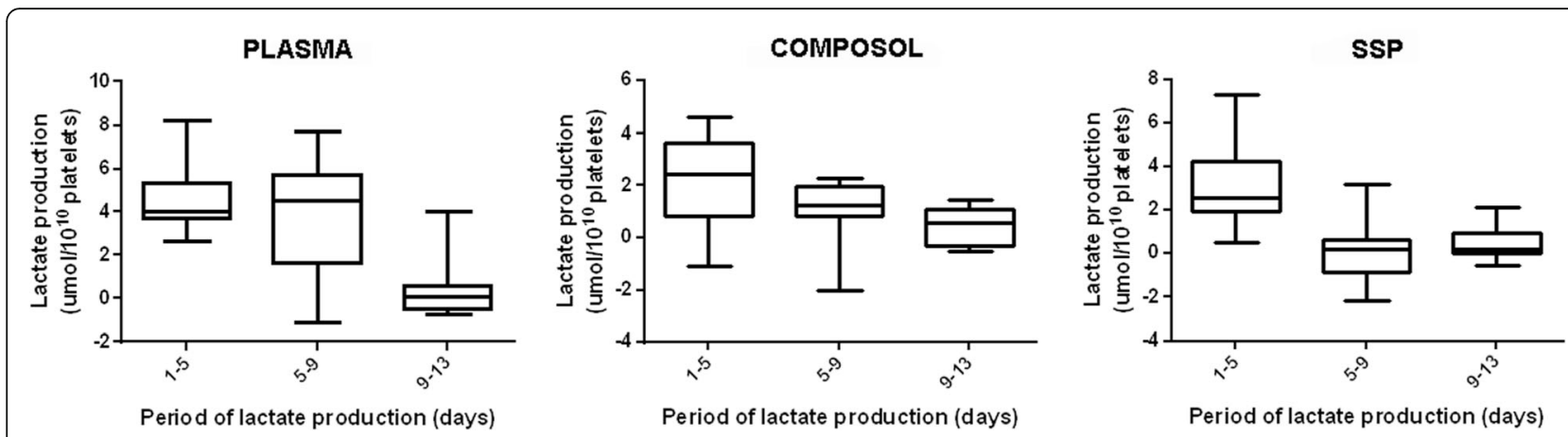

Fig. 3 Lactate production rates of platelet concentrates stored in plasma and additive solution for 13 days

viscosity of the PAS, makes it necessary to adapt and standardize the centrifugation protocols $[24,25]$.

Platelet swirling is used as a feasibility indicator to measure alterations in platelet morphology from discoid to spherical [12]. Corroborating the results of our experiment, other authors observed the maintenance of swirling with SSP+ and Composol for 7 days or longer (Table 3) [3, 11, 26].

In accordance with human PC data, alterations in MPV were not observed according to the used solution, this suggests that the change of format occurs regardless of the PAS used [7]. It is observed that the PDW in the control group has significant increase on the 13th day of storage which shows that the platelet fragmentation is more expressive in this group. These findings suggest that there is lysis and microparticle formation within the storage time [27, 28]. MPV showed a strong correlation with the $\mathrm{pH}$ decreasing over storage time, that indicate a loss of discoid to spherical shape, reflected in the MPV increase. Also we observed a strong correlation MPV and PDW with $\Delta \Psi \mathrm{m}$ decreasing suggesting mitochondrial swelling and loose of potential (Fig. 1, Table 2) [29].

As a marker of platelet surface, the results of the use of CD61 are in agreement with other studies where the percentage of expression remained independent from the PAS used and the storage time [30, 31]. The CD62P is described with inconsistent results when correlating the increased expression with post-transfusion increment $[29,32]$. In our experiment, the highest percentage of activation on day 1 was associated with the intense manipulation of the blood bag for its preparation, as described for human platelets. On day 13, CD62P expression was correlated with loss of platelet function [30].

When inferring the platelet metabolism, $\mathrm{pH}$ is one of the most used tools for quality control, turning into a mandatory assessment item at blood banks. When evaluating SSP+ and Composol with $35 \%$ of residual plasma in human $\mathrm{PC}$, the $\mathrm{pH}$ maintenance was observed $(>6.6)$ until the 8th day of storage (Table 3) [3, 30]. In agreement with previous studies, the $\mathrm{pH}$ decreased in all

Table 2 Pearson correlation between the metabolic characteristics of the stored plasma concentrates and the additive solution for 13 days

\begin{tabular}{|c|c|c|c|c|c|c|c|c|c|c|c|}
\hline Correlations & Glucose & Lactate & $\mathrm{pH}$ & $\mathrm{HCO}_{3}$ & ATP & $\mathrm{pO}_{2}$ & $\mathrm{pCO}_{2}$ & MPV & PDW & $\begin{array}{l}\Delta \psi_{\mathrm{m}} \\
(\mathrm{JC}-1)\end{array}$ & $\mathrm{LDH}$ \\
\hline Glucose & - & $-.220^{*}$ & $.468^{* *}$ & $.667^{* *}$ & $.246^{*}$ & .093 & $.338^{* *}$ & $-.283^{*}$ & $-.321^{* *}$ & $.284^{* *}$ & $-.226^{*}$ \\
\hline Lactate & $-.220^{*}$ & - & $-.663^{* *}$ & $-.590^{* *}$ & $-.285^{* *}$ & $.596^{* *}$ & $-.659^{* *}$ & $.477^{* *}$ & $.500^{* *}$ & $-.800^{* *}$ & $.463^{* *}$ \\
\hline $\mathrm{pH}$ & $.468^{* *}$ & $-.663^{* *}$ & - & $.706^{* *}$ & $.253^{* *}$ & $-.525^{* *}$ & $.542^{* *}$ & $-.549^{* *}$ & $-.455^{* *}$ & $.660^{* *}$ & $-.227^{* *}$ \\
\hline $\mathrm{HCO}_{3}$ & $.667^{* *}$ & $-.590^{* *}$ & $.706^{* *}$ & - & $.397^{* *}$ & $-.366^{* *}$ & $.695^{* *}$ & $-.507^{* *}$ & $-.526^{* *}$ & $.604^{* *}$ & -.073 \\
\hline ATP & $.246^{*}$ & $-.285^{* *}$ & $.253^{* *}$ & $.397^{* *}$ & - & $-.169^{*}$ & $.262^{* *}$ & -.160 & -.128 & $.350^{* *}$ & -.099 \\
\hline $\mathrm{pO}_{2}$ & .093 & $.596^{* *}$ & $-.525^{* *}$ & $-.366^{* *}$ & $-.169^{*}$ & - & $-.736^{* *}$ & $.256^{* *}$ & $.257^{* *}$ & $-.602^{* *}$ & $.179^{*}$ \\
\hline $\mathrm{pCO}_{2}$ & $.338^{* *}$ & $-.659^{* *}$ & $.542^{* *}$ & $.695^{* *}$ & $.262^{* *}$ & $-.736^{* *}$ & - & $-.314^{* *}$ & $-.389^{* *}$ & $.708^{* *}$ & $-.336^{* *}$ \\
\hline MPV & $-.283^{*}$ & $.447^{* *}$ & $-.549^{* *}$ & $-.507^{* *}$ & -.160 & $.256^{* *}$ & $-.314^{* *}$ & - & $.855^{* *}$ & $-.440^{* *}$ & .017 \\
\hline PDW & $-321^{* *}$ & $.500^{* *}$ & $-.455^{* *}$ & $-.526^{* *}$ & -.128 & $.257^{* *}$ & $-.389^{* *}$ & $.855^{* *}$ & - & $-.550^{* *}$ & .102 \\
\hline$\Delta \psi_{\mathrm{m}}(\mathrm{JC}-1)$ & $.284^{* *}$ & $-.800^{* *}$ & $-.660^{* *}$ & $.604^{* *}$ & $.350^{* *}$ & $-.602^{* *}$ & $.708^{* *}$ & $-.440^{* *}$ & $-.550^{* *}$ & - & $-.392^{* *}$ \\
\hline $\mathrm{LDH}$ & $-.226^{*}$ & $.463^{* *}$ & $-.277^{* *}$ & -.073 & -.099 & $.179 *$ & $-.336^{* *}$ & .017 & .102 & $-.392^{* *}$ & - \\
\hline
\end{tabular}

*Correlation with significance level $p<0.05$. ${ }^{* *}$ Correlation with significance level $p<0.01$ 


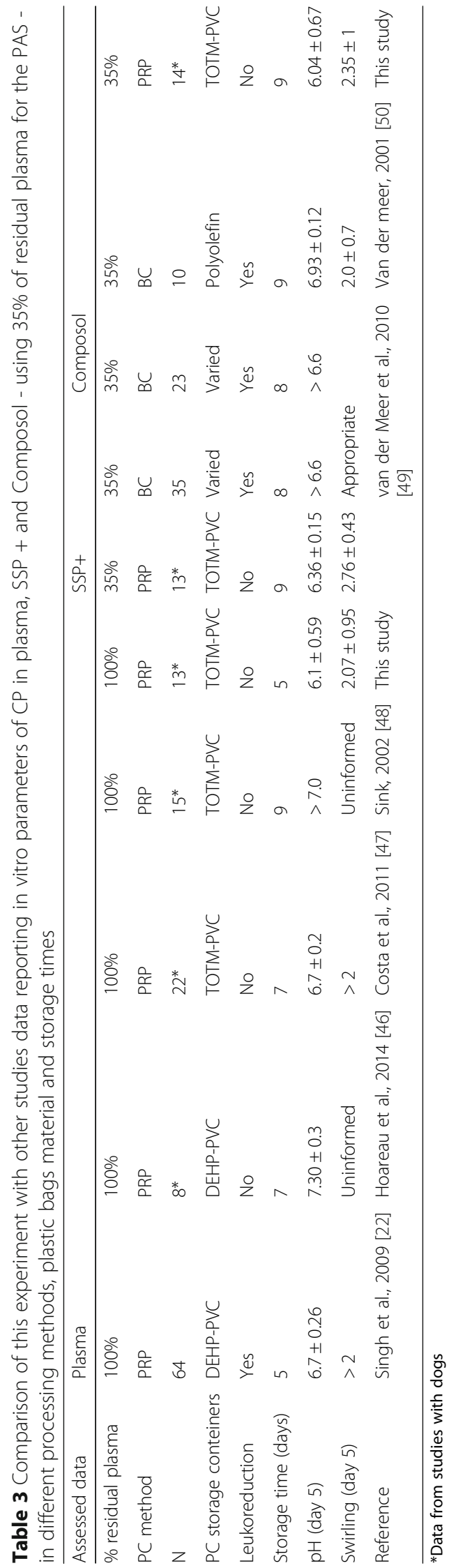


canine PC units during storage, but it remained within acceptable limits $(>6.2)$ until day 13 just in the samples stored in SSP+. In other studies, this solution obtained $\mathrm{pH}$ above 7.0 on the 7 th day of storage, using similar amounts of residual plasma (30\%) [11, 31].

The choice of TOTM-PVC material as plastic for the bags of this study was based on results of previous studies which showed better $\mathrm{pH}$ stability in canine $\mathrm{PC}$ when compared to the use of DEHP-PVC [20]. Studies using DEHP-PVC bags consequently showed a lower $\mathrm{pH}$ during storage [33, 34]. Both materials are recommended for human PC storage in Brazilian blood banks [35].

The reduction of ATP levels observed in our study was correlated to a decrease of glucose and $\Delta \Psi \mathrm{m}$ which results in the reduction of energy metabolism. When metabolically active, the platelet mitochondria produce ATP through anaerobic glycolysis which occurs in the cytosol as well as through the aerobic oxidation that occurs by the tricarboxylicacid cycle (TCA) $[8,36]$. In reference to metabolism, it is highlighted that the PAS have low amount of glucose in order to reduce the rate of anaerobic glycolysis, which can be clearly evidenced by glucose consumption and lactate production rates. In comparison to the control group, the variation in lactate production in PAS occurs because of their emphasizing the aerobic oxidation. In our study, the results of the lactate and glucose rates refer to the same behavior of human PCs evaluated in plasma or PAS [37, 38]. Glucose consumption and lactate production are also stimulated by the presence of phosphate in the storage media. Therefore, the $100 \%$ plasma group showed significantly higher conversion rate compared to Composol or SSP+. Composol, which contains no phosphate in its formulation, showed the lowest conversion rates. The phosphate is present in $\mathrm{SSP}+$, but the presence of potassium and magnesium counteracts its effect, and this provides low conversion rates similar to those observed with Composol [39]. There are no studies evaluating production and consumption rates in canine $\mathrm{PC}$, but the studies that assessed glucose and lactate concentration observed metabolic behavior and values similar to those reported in humans [20, 21].

The bicarbonate concentration is influenced by the composition of PAS [39]. It is also observed that the bicarbonate concentration was more stable in SSP+; this pattern reflects the most stable $\mathrm{pH}$ values in this $\mathrm{PC}$ group. The same behavior was observed in another study comparing $100 \%$ plasma PC with $70 \%$ SSP+ for 14 days [38]. The presence of bicarbonate in the plasma has a buffering effect and in order to compensate the absence of this component, acetate was added to PAS. In TCA, acetate is metabolized to acetyl-CoA, consuming $\mathrm{H}+$, and oxidized to $\mathrm{CO}_{2}$ and $\mathrm{H}_{2} \mathrm{O}$, acting in a buffering mode against $\mathrm{pH}$ reduction caused by glycolysis $[6,36,40]$.
A reduction in $\mathrm{pCO}_{2}$ is observed along the storage time and $\mathrm{pO}_{2}$ behaves inversely. Studies in human $\mathrm{PC}$ report an increase of $\mathrm{pO}_{2}$ and a decrease in $\mathrm{pCO}_{2}$ associated with the decline of oxidative metabolism [34]. Similar results to our study were obtained when comparing plasma to PAS $\mathrm{PC}$ in humans, and correlated $\mathrm{pCO}_{2}$ values with the bicarbonate concentration of the medium $[17,30]$.

A previous study on PSL showed that the shelf-life and viability correlates with apoptosis mechanisms [41]. Therefore, the identification of intrinsic path markers (PS exposure, caspase marking) as well as extrinsic ones (quantification of cytochrome c, $\Delta \Psi \mathrm{m}$ ) have been used. The PS exposure is considered to be an activation marker with pro-coagulant activity, and also an apoptosis indicator [1, 32]. In our study, no alterations during storage time were observed, however there is a greater expression in the control group on all the days of evaluation if compared to SSP+. In most studies, the expression percentage increases along time in $100 \%$ plasma PC and with the use of PAS, with a more significant expression in plasma $[3,38]$. One experiment evaluated in vitro canine platelets for 8 days and observed an increase of PS only after the addition of apoptosis inducing agent in the environment. Previously to the effect of the inducing agent, the expression percentage was similar to that observed in canine PC from our study [42]. Our experiment partially reproduces the behavior observed in human PC, although there is no increase over time, we observed a greater expression in the $100 \%$ plasma group. This scenario may reflect a limitation of the technique used in dogs; or the fact that the exposure of PS is not a good marker of recent apoptosis in canine platelets.

LDH is a marker of senescence. As the metabolic potential of platelets is exhausted, platelet lysis occurs as well as there lease of cytosolic components including LDH $[10,32]$. Studies in canine and human plasma PC and the use of PAS in human PCs demonstrate results that corroborate with those observed in our experiment $[19,20,30]$. This mechanism also reflects the positive results of Pearson correlation with lactate and negative results with $\mathrm{pH}$ and $\Delta \Psi \mathrm{m}$ (Fig. 1, Table 2).

The assessment of mitochondrial potential is most commonly used when the goal is to assess interference of mitochondria in storage time or situations when the $\mathrm{PC}$ is not kept under constant stirring. There is few data concerning mitochondrial function and platelet viability with the use of PAS $[29,43]$. The results presented here are in accordance with the evaluation of this parameter using SSP+ in human PC [38] and a single study in canine plasma PC (Lasta C.: Metabolism of canine platelets stored as platelet concentrate for 5 days, unpublished). Another group assessed Composol in human PC, observing mitochondrial viability from 12 days of storage, the results were consistent with our study; where there 
is a drop of $\Delta \Psi \mathrm{m}$ from the 9th day, which is more significant in the PC control group [10].

The bacterial contamination of 3 units was an obstacle. Coagulase negative Staphylococcus usually originates from the donor's skin and is often isolated in PC; and when it is transfused, it can cause fatal reactions associated with endotoxemia. Because of the intense manipulation, the care must be redoubled when the additive solution is added in the processing [5]. Two of the excluded units were from donors in which the owner did not allow trichotomy. The authors suggest that in these cases, the bags do not be used for CP production. However, it was not possible to determine if the contamination occurred at the time of the blood collection or during the sampling procedure of the blood bags. The contaminated samples were excluded from the study.

\section{Correlations}

The correlations enabled the identification of the main tendency among the variables used for platelet evaluation during storage. Platelet metabolism requires some direct correlations such as those between glucose and lactate, where the consumption of glucose directly results in lactate production, as well as between $\mathrm{pH}$ and $\mathrm{pCO}_{2}$, where the $\mathrm{pH}$ was negatively correlated with $\mathrm{pCO}_{2}$. Furthermore, the relationship between $\mathrm{pO}_{2}$ and $\mathrm{pH}$ suggests that oxygen availability is strongly associated with maintenance of $\mathrm{pH}$ in an osmotic balanced environment. Some correlations are indirect, but they are intuitive between glucose/lactate and morpho-structural rates (i.e. MPV, PDW), apoptotic markers (i.e. LDH) and $\Delta \Psi \mathrm{m}$. These parameters represent the measure of platelet viability; low glucose or no glucose is usually the result of prolonged storage, which indirectly results in loss of viability. The main cause for the consumption of glucose, besides the regular metabolism, is the constant energy requirement to maintain the osmotic balance and the integrity of the platelet membrane, lost over time. This metabolic shift to aerobic glycolysis is probably responsible for the observed correlations between glucose and lactate (Warburg effect). Immune cells, as platelets, switch their energy production from oxidative phosphorylation to glycolysis upon cell activation. This metabolic programming has been attributed to facilitate cytoskeletal changes, increased ion signaling, enhanced phospholipid turnover, and greater macromolecule synthesis in a very short time during platelet activation [44]. There is a strong correlation among glucose, lactate, MPV, LDH and $\triangle \Psi \mathrm{m}$ which indicates that the metabolic rate is related to degranulation, osmotic destabilization and/or swelling.

\section{Conclusions}

$\mathrm{SSP}+$ and Composol seem to be an excellent alternative to replace plasma in the production of canine PC.
Based on in vitro variables, canine PC storage in Composol is viable for 9 days, whereas the viability extends to 13 days with the use of SSP+. Prolonged storage, with in vitro quality maintenance, seems to be possible in veterinary medicine, with results similar to those seen in human platelets.

In vitro studies do not necessarily predict posttransfusion efficacy. Therefore, in vivo studies are needed to estimate the real contribution of PAS in preventing the PSL. This study provides a technical point of view needed for canine PC production with low plasma concentrations, and addition of PAS.

\section{Endnotes \\ ${ }^{1}$ CPD SAGM, Standard Handbags, JP Pharmaceuticals, $\mathrm{BR}$ \\ ${ }^{2}$ PASIIIM, PAS-E, Macopharma, FR \\ ${ }^{3}$ PAS-D, Fresenius Kabi, NLD \\ ${ }^{4}$ Sorvall Legend RT centrifugal, Thermo Scientific, US \\ ${ }^{5}$ ACS201, Terumo Medical do Brazil, BR \\ ${ }^{6}$ Fenwal, Fresenius Kabi, US \\ ${ }^{7} \mathrm{CDCI} 3$, Indrel, BR \\ ${ }^{8}$ AP48L, Presvac, AR \\ ${ }^{9}$ LO-Laboroptik GmbH, DE \\ ${ }^{10}$ Poch-100iV Diff, Roche Diagnostics Brazil, BR \\ ${ }^{11}$ clone VI-PL2, BD Biosciences, US \\ ${ }^{12}$ CD62P, AC1.2 clone, BD Biosciences, US \\ ${ }^{13}$ CG8, ISTAT Abbott Point of Care, CA \\ ${ }^{14}$ Lyte Luminescence ATP detection 1,010,224, Perkin Elmer, NL \\ ${ }^{15}$ SpectramaxM5, Molecular Devices Inc., US \\ ${ }^{16}$ Vitros 250 Chemistry System, Ortho Clinical Diagnos- tics, Johnson \& Johnson, BR \\ ${ }^{17} \mathrm{HI}$ 9126, Hanna Instruments Inc., US \\ ${ }^{18}$ ApoFlowEx FITC Kit, Exbio, CZ \\ ${ }^{19}$ MitoScreen JC-1, BD Biosciences, US \\ ${ }^{20}$ FACSCalibur $^{\mathrm{Tm}}$ and CellQuest ${ }^{\mathrm{TM}}$ Pro software, BD Bio- sciences, US \\ ${ }^{21}$ De Novo, Software, US \\ ${ }^{22}$ GraphPad Software, US.}

\section{Additional files}

Additional file 1: Composition of the solutions used in the experiment. (DOCX 14 kb)

Additional file 2: Platelet count. $\mathrm{pH}$ and CD61 percentage of positive cells mean \pm standard deviation. Different lowercase letters represent significantly different values $(p<0.05)$ between treatments. Different symbols represent significantly different values $(p<0.05)$ between assessment days. (DOCX $15 \mathrm{~kb}$ )

Additional file 3: Glucose consumption mean \pm standard deviation of platelet concentrates stored in plasma and additive solution for 13 days. Different lowercase letters represent significantly different values $(p<0.05)$ between treatments. Different symbols represent significantly different values $(p<0.05)$ between assessment days. (DOCX $13 \mathrm{~kb}$ ) 
Additional file 4: Lactate production mean \pm standard deviation of platelet concentrates stored in plasma and additive solution for 13 days. Different lowercase letters represent significantly different values $(p<0.05)$ between treatments. Different symbols represent significantly different values $(p<0.05)$ between assessment days. (DOCX $14 \mathrm{~kb}$ )

\section{Abbreviations}

ATP: Adenosine-5'-triphosphate; BC: Buffy-coat method; BHI: Brain-heart infusion medium; CBC: Complete blood count; CD61: Monoclonal antibodies (MoAbs) for GPIb expression; CD62P: p Selectin expression; CPD: Citrate phosphate dextrose; DEHP-PVC: Di(2-ethylhexyl)phthalate softener for polyvinyl chloride (PVC); FITC: Fluorescein isothiocyanate; JC-1: J-aggregate forming cation; LDH: Lactic acid dehydrogenase; MPV: Mean platelet volume; PAS: Platelet additive solutions; PC: Platelet concentrate; PDW: Platelet distribution width; PRP: Platelet rich plasma method; PS: Phosphatidylserine, annexin V marker; PSL: Platelet storage lesions; TCA: Tricarboxylic acid cycle; TOTM-PVC: Tri(2-Ethylhexyl) Trimellitate softener for polyvinyl chloride (PVC); $\Delta \psi \mathrm{m}$ : Mitochondrial membrane potential

\section{Acknowledgements}

The authors would like to acknowledge Sanquin Blood Bank (Department R\&D, Amsterdam, NL) for the technical support in the use of additive solutions. Likewise, we acknowledge Blut's Diagnostic Center and Veterinary Services (Porto Alegre, RS, Brazil) for allowing the use of its physical structure for collection and processing of blood components.

\section{Funding}

This study was financed by the National Research Council (CNPq, Brazil). The role of the funding body in the design of the study was to provide acquisition of reagents and consumables for research. At the end of the research, a report was sent with the results obtained.

\section{Availability of data and materials}

The datasets used and/or analysed during the current study are available from the corresponding author on reasonable request.

\section{Authors' contributions}

$\mathrm{NH}, \mathrm{CSL}$ planned and suited the project, performed the standardization of the tests, evaluation of donors and collection of blood bags, participated in experimental analysis, transcription and data analysis, were the major contributor in writing the manuscript. MLD, LAL standardized of biochemical and spin testing, evaluated of donors and collection of blood bags, substantial contributions to conception and design of the experiment, performed the evaluation of donors, interpretation of data. NAM, SRT, FBF standardized flow cytometry experiments, take part in experimental analysis, transcription, involved in drafting and critically revising the manuscript. DK, FHDG provide substantial contributions to conception and design of the experiment, interpretation of data, revising the manuscript. All authors were involved in drafting manuscript, ensure the accuracy and integrity of the data and give final approve to the version to be published.

\section{Ethics approval and consent to participate}

The animals were included in the study after the tutor's permission, and in accordance with bioethics concepts applied to animal research [45]. This study was approved by the Ethics Committee on Animal Use from the Federal University of Rio Grande do Sul (Approval protocol \#20528).

\section{Consent for publication}

Not applicable

\section{Competing interests}

The authors declare that they have no competing interests.

\section{Publisher's Note}

Springer Nature remains neutral with regard to jurisdictional claims in published maps and institutional affiliations.

\section{Author details}

${ }^{1}$ Clinical Pathology Laboratory, Veterinary Medicine Faculty, Universidade do Sul de Santa Catarina, Tubarão, Brazil. ${ }^{2}$ Veterinary Medicine Faculty, Centro
Universitário Ritter dos Reis - Laureatte International Universities, Porto Alegre, Brazil. 'Blut's Diagnosis Center and Veterinary Services, Porto Alegre, Brazil. ${ }^{4}$ Sanquin Blood Bank and Sanquin Research, Sanquin, Amsterdam, the Netherlands. ${ }^{5}$ Post-Graduation Program in Medicine: Medical Sciences, Universidade Federal do Rio Grande do Sul, Porto Alegre, Brazil. ${ }^{6}$ Zanol Laboratory, Porto Alegre, Brazil. 'DDepartment of Veterinary Clinical Pathology, Universidade Federal do Rio Grande do Sul, Porto Alegre, Brazil.

Received: 24 April 2017 Accepted: 30 October 2017

Published online: 15 November 2017

\section{References}

1. Dasgupta SK, Argaiz ER, Mercado JE, Maul HO, Garza J, Enriquez AB, AbdelMonem H, Prakasam A, Andreeff M, Thiagarajan P. Platelet senescence and phosphatidylserine exposure. Transfusion. 2010;50(10):2167-75.

2. Heaton WA. Costs and benefits of PAS platelets: a mix of science, quality, and value. Transfusion. 2013;53(11):2597-602.

3. Leitner GC, List J, Horvath M, Eichelberger B, Panzer S, Jilma-Stohlawetz P. Additive solutions differentially affect metabolic and functional parameters of platelet concentrates. Vox Sang. 2015;110(1):20-26.

4. Nguyen TC, Cruz MA, Carcillo JA. Thrombocytopenia-associated multiple organ failure and acute kidney injury. Crit Care Clin. 2015;31(4):661-74.

5. Kreuger AL, Middelburg RA, Kerkhoffs JH, Schipperus MR, Wiersum-Osselton $J$ C, van der Bom JG. Storage medium of platelet transfusions and the risk of transfusion-transmitted bacterial infections. Transfusion. 2017;57(3):657-60.

6. Shimizu M, Ando Y, Takei M, Miyachi H, Tanaka Y, Kawada T. Whole blood flow cytometry for detection of activated platelets. I. Platelet identification, blood collection and storage. Rinsho Byori. 2001;49(4):402-7.

7. Leytin V, Allen DJ, Mutlu A, Mykhaylov S, Lyubimov E, Freedman J. Platelet activation and apoptosis are different phenomena: evidence from the sequential dynamics and the magnitude of responses during platelet storage. Br J Haematol. 2008;142:494-7. England

8. van der Meer PF. PAS or plasma for storage of platelets? A concise review. Transfus Med. 2016;26(5):339-42.

9. Kaufman RM, Djulbegovic B, Gernsheimer T, Kleinman S, Tinmouth AT, Capocelli KE, Cipolle MD, Cohn CS, Fung MK, Grossman BJ, et al. Platelet transfusion: a clinical practice guideline from the AABB. Ann Intern Med. 2015;162(3):205-13.

10. Amanat ST, Shakoor HA, Raza M, Khan N, Rauf A. Clinical indications and adverse reactions of platelet apheresis. J Coll Physicians Surg Pak. 2015:25(6):403-6.

11. Gravemann U, Volgmann T, Min K, Philipp R, Lambrecht B, Müller TH, Seltsam A. In vitro variables of buffy coat-derived platelet concentrates with residual plasma of down to $10 \%$ are stably maintained in new-generation platelet additive solutions. Transfusion. 2015;55(7):1700-9.

12. Johnson L, Loh YS, Kwok M, Marks DC. In vitro assessment of buffy-coat derived platelet components suspended in SSP+ treated with the INTERCEPT blood system. Transfus Med. 2013;23(2):121-9.

13. Wagner SJ, Myrup A, Awatefe H, Thompson-Montgomery D, Hirayama J, Skripchenko A. Maintenance of platelet in vitro properties during 7-day storage in $\mathrm{M}$-sol with a 30-hour interruption of agitation. Transfusion. 2008;48(12):2501-607.

14. Moghimi SM, Hunter AC, Peer D. Platelet mimicry: the emperor's new clothes? Nanomedicine. 2015:12(1):245-8.

15. Nair CS, Vidya R, Ashalatha PM. Hexamoll DINCH plasticised PVC containers for the storage of platelets. Asian J Transfus Sci. 2011;5(1):18-22.

16. ANVISA: Boas práticas no ciclo do sangue. In: RDC N ${ }^{\circ} 34$. Edited by ANVISA, vol. RDC Nº 34. Brasil: Diário Oficial da União, 11 DE JUNHO DE 201; 2014: 39.

17. Bhal V, Herr MJ, Dixon M, Akins S, Hord E, White MM, Seiffert D, Kotha J, Jennings LK. Platelet function recovery following exposure to triple antiplatelet inhibitors using an in vitro transfusion model. Thromb Res. 2015; 136(6):1216-23.

18. Sweeney J, Kouttab N, Holme S, Kurtis J, Cheves T, Nelson E. Storage of platelet-rich plasma-derived platelet concentrate pools in plasma and additive solution. Transfusion. 2006:46(5):835-40.

19. Meira Martins LA, Vieira MQ, Ilha M, de Vasconcelos $M$, Biehl HB, Lima DB, Schein V, Barbe-Tuana F, Borojevic R, Guma FC. The interplay between apoptosis, mitophagy and mitochondrial biogenesis induced by resveratrol can determine activated hepatic stellate cells death or survival. Cell Biochem Biophys. 2015;71(2):657-72. 
20. Abela GS, Huang R, Ma H, Prieto AR, Lei M, Schmaier AH, Schwartz KA, Davis JM. Laser-light scattering, a new method for continuous monitoring of platelet activation in circulating fluid. J Lab Clin Med. 2003;141(1):50-7.

21. Snyder EL, Bookbinder M, Kakaiya R, Ferri P, Kiraly T. 5-day storage of platelet concentrates in CLX containers: effect of type of agitation. Vox Sang. 1983:45(6):432-7.

22. Singh RP, Marwaha N, Malhotra P, Dash S. Quality assessment of platelet concentrates prepared by platelet rich plasma-platelet concentrate, buffy coat poor-platelet concentrate (BC-PC) and apheresis-PC methods. Asian J Transfus Sci. 2009;3(2):86-94.

23. Clemmons RM, Meyers KM. Acquisition and aggregation of canine blood platelets: basic mechanisms of function and differences because of breed origin. Am J Vet Res. 1984;45(1):137-44.

24. Morison IM, Cramer Borde EM, Cheesman EJ, Cheong PL, Holyoake AJ, Fichelson S, Weeks RJ, Lo A, Davies SM, Wilbanks SM, et al. A mutation of human cytochrome $c$ enhances the intrinsic apoptotic pathway but causes only thrombocytopenia. Nat Genet. 2008:40(4):387-9.

25. van der Meer, PF. PAS or plasma for storage of platelets? A concise review. Transfusion Medicine. 2016;26(5):339-42.

26. Leitner GC, List J, Horvath M, Eichelberger B, Panzer S, Jilma-Stohlawetz P. Additive solutions differentially affect metabolic and functional parameters of platelet concentrates. Vox Sang. 2016;110(1):20-6.

27. Helmond SE, Catalfamo JL, Brooks MB. Flow cytometric detection and procoagulant activity of circulating canine platelet-derived microparticles. Am J Vet Res. 2013;74(2):207-15.

28. Mobarrez F, Antovic J, Egberg N, Hansson M, Jorneskog G, Hultenby K, Wallen $\mathrm{H}$. A multicolor flow cytometric assay for measurement of plateletderived microparticles. Thromb Res. 2010;125(3):e110-6.

29. Tong S, Wang H, Zhang T, Chen L, Liu B. Accumulation of CD62P during storage of apheresis platelet concentrates and the role of CD62P in transfusion-related acute lung injury. Mol Med Rep. 2015;12(5): 7777-81.

30. Vassallo RR, Adamson JW, Gottschall JL, Snyder EL, Lee W, Houghton J, Elfath MD. In vitro and in vivo evaluation of apheresis platelets stored for 5 days in 65\% platelet additive solution/35\% plasma. Transfusion. 2010;50(11):2376-85.

31. Sodergren AL, Tynngard N, Berlin G, Ramstrom S. Responsiveness of platelets during storage studied with flow cytometry - formation of platelet subpopulations and LAMP-1 as new markers for the platelet storage lesion. Vox Sang. 2015; 110 (2): 116-25.

32. Zhuang Y, Ren G, Li H, Tian K, Zhang Y, Qiao W, Nie X, Liu Y, Song Y, Zhu C. In vitro properties of apheresis platelet during extended storage in plasma treated with anandamide. Transfus Apher Sci. 2014;51(1):58-64.

33. Tynngard N, Lindahl TL, Trinks M, Studer M, Berlin G. The quality of platelet concentrates produced by COBE spectra and Trima Accel cell separators during storage for 7 days as assessed by in vitro methods. Transfusion. 2008;48(4):715-22.

34. Prowse CV, de Korte D, Hess JR, van der Meer PF. Commercially available blood storage containers. Vox Sang. 2014;106(1):1-13.

35. ANVISA ANdVS: Ensaiospara bolsas plásticas para coleta, armazenamento e transferência de sanque humano e seus componentes. In: RDC $N^{\circ} 35$. Edited by ANVISA, vol. RDC N 35. Brasil: Diário Oficial da União, 12 de Junho de 2014; 2014: 22

36. Chandra T, Gupta A, Kumar A. Extended shelf life of random donor platelets stored for 7 days in platelet additive solution at different temperatures. Biom J. 2014;37(4):211-7.

37. Diedrich B, Sandgren P, Jansson B, Gulliksson H, Svensson L, Shanwell A. In vitro and in vivo effects of potassium and magnesium on storage up to 7 days of apheresis platelet concentrates in platelet additive solution. Vox Sang. 2008;94(2):96-102.

38. Pati HP, Jain S. Flow cytometry in hematological disorders. Indian J Pediatr. 2013;80(9):772-8.

39. Skripchenko A, Turgeon A, Thompson-Montgomery D, Awatefe $H$, Wagner SJ. Value of calcium and phosphate in a bicarbonate-containing platelet additive solution with low plasma levels in maintaining key in vitro platelet storage parameters. Transfusion. 2017:57(2):349-56

40. Albanyan AM, Harrison P, Murphy MF. Markers of platelet activation and apoptosis during storage of apheresis- and buffy coat-derived platelet concentrates for 7 days. Transfusion. 2009;49(1):108-17.

41. Saboor M, Moinuddin M, llyas S. New horizons in platelets flow cytometry. Malays J Med Sci. 2013;20(2):62-6.

42. Pereira J, Soto M, Palomo I, Ocqueteau M, Coetzee LM, Astudillo S, Aranda E, Mezzano D. Platelet aging in vivo is associated with activation of apoptotic pathways: studies in a model of suppressed thrombopoiesis in dogs. Thromb Haemost. 2002;87(5):905-9.

43. Mathai J, Resmi KR, Sulochana PV, Sathyabhama S, Baby Saritha G, Krishnan LK. Suitability of measurement of swirling as a marker of platelet shape change in concentrates stored for transfusion. Platelets. 2006;17(6):393-6.

44. Delmastro-Greenwood MM, Piganelli JD. Changing the energy of an immune response. Am J Clin Exp Immunol. 2013;2(1):30-54.

45. Rinder HM, Smith BR. In vitro evaluation of stored platelets: is there hope for predicting posttransfusion platelet survival and function? Transfusion. 2003;43:2-6. United States

46. Hoareau GL, Jandrey KE, Burges J, Bremer D, Tablin F. Comparison of the platelet-rich plasma and buffy coat protocols for preparation of canine platelet concentrates. Veterinary Clinical Pathology. 2014;43(4):513-18.

47. Costa, CCMR. Influências das alterações físicas e químicas sobre a função plaquetária e a expressão da GPIllbllla em concentrados de plaquetas caninos estocados (Masters in Science in Veterinary Medicine). Veterinary Medicine and Zootechny Faculty, Universidade do Estadual Paulista. 2011; 91 p.: Botucatu, Sao Paulo, Brazil.

48. Sink, C. Canine Platelet Concentrates: An In Vitro Study to Effectively Provide a Source of Functional Platelets. 2002. 67 (Master of Science in Veterinary Medical Science). Virginia Polytechnic Institute. 2002; 67p.: Virginia, United States.

49. van der Meer PF, Kerkhoffs JL, Curvers J, Scharenberg J, de Korte D, Brand A, de Wildt-Eggen J. In vitro comparison of platelet storage in plasma and in four platelet additive solutions, and the effect of pathogen reduction: a proposal for an in vitro rating system.Vox Sanguinis. 2010:98(4):517-24

50. van der Meer PF, Pietersz RNI, Reesink HW. Comparison of two platelet additive solutions. Transfusion Medicine. 2001;11(3):193-97.

\section{Submit your next manuscript to BioMed Central and we will help you at every step:}

- We accept pre-submission inquiries

- Our selector tool helps you to find the most relevant journal

- We provide round the clock customer support

- Convenient online submission

- Thorough peer review

- Inclusion in PubMed and all major indexing services

- Maximum visibility for your research

Submit your manuscript at www.biomedcentral.com/submit
C) Biomed Central 